\title{
ON TRIGONOMETRICAL SERIES WHOSE COEFFICIENTS DO NOT TEND TO ZERO $^{1}$
}

\author{
R. SALEM
}

Let $\sum \rho_{n} \cos \left(n x-\alpha_{n}\right)$ be a series $(S)$ such that

$$
\rho_{n} \geqq 0, \quad \lim \sup \rho_{n}>0 .
$$

It is well known that the set $E$ of convergence of $S$ is of measure zero, a result due to Cantor and Lebesgue. More recently Rajchman has proved that $E$ is a sum of an enumerable sequence of $H$-sets, and so in particular (an $H$-set being closed and of measure zero) that $E$ is of the first category. ${ }^{2}$

We propose to establish a more precise property of the sets $E$ and to show the connection between these sets and sets of absolute convergence (that is, sets in which a trigonometrical series can converge absolutely without being absolutely convergent everywhere).

We propose to call, in memory of Rajchman, "set of the type $R$ " any set $E$ such that a series $(S)$ exists which satisfies the condition (1) and converges in $E$. $E$ being a sum of closed sets it is natural to investigate the properties of perfect sets of the type $R$. Let $P$ be such a set. For every $x$ belonging to $P$ we must have $\lim \rho_{n} \cos \left(n x-\alpha_{n}\right)=0$. But by (1) there exists an infinite sequence of integers $\left\{n_{k}\right\}$ such that $\rho_{n_{k}}>r>0$. Hence,

$$
\lim \cos \left(n_{k} x-\alpha_{n_{k}}\right)=0 .
$$

We can assume, without loss of generality that the point $x=0$ belongs to $P$. Hence

$$
\lim \cos \alpha_{n_{k}}=0 .
$$

(2) and (3) give immediately

$$
\lim \left|\sin n_{k} x \sin \alpha_{n_{k}}\right|=0,
$$

and as by (3)

$$
\lim \left|\sin n_{k} x \cos \alpha_{n_{k}}\right|=0
$$

we get, by adding (4) and (5),

$$
\lim \left|\sin n_{k} x\right|=0 .
$$

\footnotetext{
1 Presented to the Society, May 2, 1941.

${ }^{2}$ See Zygmund, Trigonometrical Series, Warsaw, 1935, pp. 267-270.
} 
This implies, for every $x$ belonging to $P, \lim \sin ^{2} n_{k} x=0$. Let $F(x)$ be any non-decreasing bounded function, constant in each interval contiguous to $P$, but not everywhere. The sequence $\left\{\sin ^{2} n_{k} x\right\}$ being uniformly bounded, we have

$$
\lim \int_{0}^{2 \pi} \sin ^{2} n_{k} x d F=0
$$

or

$$
\lim \int_{0}^{2 \pi} \cos 2 n_{k} x d F=F(2 \pi)-F(0),
$$

that is to say, not only the Fourier-Stieltjes cosine coefficients of rank $2 n_{k}$ of $d F$ do not tend to zero (even if $F$ is continuous), but they tend to the greatest possible limit as $k \rightarrow \infty$. It is interesting to observe that the sequence $\left\{n_{k}\right\}$ is independent of the function $F$.

It has been proved ${ }^{3}$ that if a perfect set $P$ is a set of absolute convergence, then for every function $F$ of the above-described type

$$
\lim \sup \int_{0}^{2 \pi} \cos 2 n x d F=F(2 \pi)-F(0) .
$$

Thus it appears that sets of the type $R$ and sets of absolute convergence are closely connected.

The necessary condition (7) shows that the condition that a set of the type $R$ should be a sum of $H$-sets is too lax. In particular, even a single $H$-set is not, in general, of the type $R$, as can be seen by the example of Cantor's ternary set $C$. This is an easy consequence of the necessary condition (6) and of the well known fact that the set of all possible sums $x+y$ where $x$ and $y$ belong to $C$ fills the whole interval on which $C$ is constructed. ${ }^{4}$

Moreover, the sum of two perfect sets of the type $R$ may not be of the type $R$. This result which has been proved by Marcinkiewic $z^{5}$ for perfect sets of absolute convergence holds good for sets of the type $R$ and is a consequence of Marcinkiewicz's argument and of the necessary condition (6).

Let now $P$ be a perfect set such that the condition (7) is satisfied. We can find a function $F$, bounded, constant in every interval con-

\footnotetext{
${ }^{3}$ See Salem, The absolute convergence of trigonometrical series, Duke Mathematical Journal, vol. 8 (1941), pp. 317-334.

${ }^{4}$ See Zygmund, loc. cit., p. 133.

5 Travaux de la Société des Sciences et des Lettres de Wilno, Classe des Sciences Mathématiques et Naturelles, vol. 12 (1937).
} 
tiguous to $P$, but increasing from one interval to another, and a sequence $\left\{m_{k}\right\}$ such that $\int_{0}^{2 \pi} \sin ^{2} m_{k} x d F<1 / k^{4}$. Hence

$$
\sum_{1}^{\infty} k^{2} \int_{0}^{2 \pi} \sin ^{2} m_{k} x d F<\infty .
$$

Hence "almost everywhere" in $P$, that is to say, in a subset $P_{1}$ of $P$ such that the variation of $F$ over $P-P_{1}$ is zero, $\sum_{1}^{\infty} k^{2} \sin ^{2} m_{k} x<\infty$. But

$$
\sum_{1}^{k}\left|\sin m_{k} x\right|<\left(\sum_{1}^{k} \frac{1}{k^{2}}\right)^{1 / 2}\left(\sum_{1}^{k} k^{2} \sin ^{2} m_{k} x\right)^{1 / 2} ;
$$

hence $\sum\left|\sin m_{k} x\right|$ converges in $P_{1}$, and $P$ is "almost everywhere" of the type $R$ (and also, almost everywhere, a set of absolute convergence).

Montreal, Canada

\section{UNITARY SPACES WITH CORRESPONDING GEODESICS ${ }^{1}$}

\section{N. COBURN}

1. Introduction. This paper is divided into three parts. In the first section, the notation and fundamental concepts of hermitian geometry are reviewed. The second section develops the equations of geodesic curves $X_{1}$ which depend on a real parameter $(t)$ and which are imbedded in a unitary space of $n$-dimensions $K_{n}$. Our principal result is: The equations of such geodesics differ from the equations of geodesics in Riemannian space in that the former contain the torsion affinor. In the third section, we classify the connections of two unitary spaces $K_{n},{ }^{\prime} K_{n}$ whose geodesics correspond. First, we find the necessary and sufficient conditions that two unitary spaces $K_{n},{ }^{\prime} K_{n}$ both with symmetric connection shall have their geodesics in correspondence. This last problem is solved in exactly the same manner as the similar problem in Riemannian space. ${ }^{2}$ Secondly, we prove that if $K_{n}$ has torsion and ' $K_{n}$ has no torsion (symmetric connection), then their geodesics do not correspond. The problem of determining all connections of unitary spaces $K_{n},{ }^{\prime} K_{n}$ both with torsion whose geodesics correspond is left open.

1 Presented to the Society, January 1, 1941.

${ }^{2}$ L. P. Eisenhart, Riemannian Geometry, Princeton University Press, 1926, p. 131. 